\title{
A discrete control algorithm synthesis for the live-steam-line heating system
}

\author{
Dmitriy Akimenko ${ }^{1}$, Alexandr Sizov, ${ }^{2, *}$, Fedor Chubarov $^{3}$, Aleksey Nikitin ${ }^{4}$, and Lusia del \\ Sokorro $^{5}$ \\ ${ }^{1}$ Bauman Moscow state technical University, IUK department, 248000 Bazhenova st., 2, Kaluga, \\ Russia \\ ${ }^{2}$ OJSC Kaluga turbine works, Designer Department, 248010 Moskovskaya st., 241k1, Kaluga, Russia \\ ${ }^{3}$ Russian agrarian University - Moscow state agricultural Academy named after K. A. Timiryazev, \\ Study Department, 248007 Vishnevskogo st., 27, Kaluga, Russia \\ ${ }^{4}$ State Autonomous professional educational institution "Kaluga technical College», Administration, \\ 248009 Grabcevskoye hw., 126, Kaluga, Russia \\ ${ }^{5}$ Don State Technical University, magistracy, 344000 Gagarin square, 1, Rostov-on-Don, Russia
}

\begin{abstract}
An original mathematic model of a main live-steam-line heating system for the $6 \mathrm{MW}$ turbine is reviewed. The present model is composed from a system of ODE with divergences and constant coefficients, along with an object structure is shown, numerical values of parameters are determined. The transfer function method is used for the model analysis, which is necessary for the main problem resolving. It consists of a discrete control algorithm construction to provide an appropriate heat transition process of an aperiodic type with quality performance required. The control algorithm is based on the functional minimization condition, which is used here as the minimum energy speed up the process. The gradient method and etalon-model method are also used to get an optimal system stage. Due to methods applied, a main variable parameter influence on a system performance is analyzed, optimal adjustment value is retrieved, which allows you to provide the required quality for the heating system performance in all operating modes.
\end{abstract}

\section{Introduction}

One of the ways for steam turbine efficiency increase is its commissioning time reduction after repair or a long downtime. As a rule, during this time the turbine equipment has enough time to cool down, so the thermal state is changed greatly.

Traditional thermal schemes of thermal power plants and nuclear power plants with steam turbine installations, as a rule, have a main steam pipeline, through which a live steam from the steam generator is supplied to the turbine [1].

Due to turbine operation manual, the startup process after a long downtime period required a smooth heating for the main steam pipeline before the same for the turbine itself.

\footnotetext{
* Corresponding author: axelxd@yandex.ru
} 
Otherwise (in case of quick injection of a large amount live steam in it) the destructive hydraulic shocks will definitely take place in pipeline [2].

Such a heating is usually provided by step-by-step or partial live steam bypass through the main pipeline to the drop. Herewith the speed and intensity of this bypass can be varied and should be optimally controlled by relevant control system. The control algorithm for this system is the object of investigation below. A thermal network of a power station, based on $6 \mathrm{MW}$ steam turbine, is considered as a task object $[1,2]$.

\section{Problem statement}

Consider the control object - a system for heating a main steam pipeline, which consists of three main elements: an electric drive with a control valve, whose task is to change the supply of a live steam to the steam line at every moment; a steam pressure sensor and the steam line itself.

The mathematical model of an object can be represented by the following system of differential equations with divergences [2]:

$$
\left\{\begin{array}{l}
T_{1} \cdot \frac{d}{d t} \rho_{n}(t)+\rho_{n}(t)=A_{1} \cdot \mu(t)+T_{1} \cdot \frac{d}{d t} \tau_{n}(t) \\
T_{2} \cdot \frac{d}{d t} \tau_{n}(t)+\tau_{n}(t)=A_{2} \cdot \mu(t)+b_{4} \cdot \tau_{c m}(t)+b_{5} \cdot \rho_{n}(t)+T_{3} \cdot \frac{d}{d t} \rho_{n}(t) \\
T_{M} \cdot \frac{d}{d t} \tau_{c m}(t)+\tau_{c m}(t)=b_{6} \cdot \tau_{n}(t) \\
T_{\ni n} \cdot \frac{d^{2}}{d t^{2}} \mu(t)+\frac{d}{d t} \mu(t)=a_{3} \cdot y(t) \\
T_{\partial} \cdot \frac{d}{d t} \Psi_{1}(t)+\Psi_{1}(t)=\rho_{n}(t)
\end{array}\right.
$$

Here $\rho_{n}(t)$ - change of an absolute steam pressure in pipeline; $T_{\partial}$ - time constant describing the inertial properties of the pressure sensor; $\mu(t)$ - the value of the relative position of the control valve (the value of the valve opening); $a_{3}=\frac{1}{T_{n x}}, T_{n x}$ - valve full stroke time; $T_{\ni n}$ - time constant of acceleration (run-out) of the output shaft of the electric drive; $\tau_{n}(t)$ - relative change in steam temperature in the steam line; $\tau_{c m}(t)$ - relative temperature change of the steam line itself.

The numerical values of the parameters above:

$$
\begin{gathered}
T_{\partial}=0,5[\mathrm{sec}] ; T_{n x}=24[\mathrm{sec}] ; T_{\text {эп }}=0,24[\mathrm{sec}] ; \\
A_{1}=3,064 ; A_{2}=2,426 \cdot 10^{-1} ; \\
b_{4}=7,362 \cdot 10^{-1} ; b_{5}=4,025 \cdot 10^{-3} ; b_{6}=1 ; \\
T_{1}=3,778[\mathrm{sec}] ; T_{2}=8,823 \cdot 10^{-1}[\mathrm{sec}] ; T_{3}=1,342 \cdot 10^{-2}[\mathrm{sec}] ; T_{M}=4,858 \cdot 10^{1}[\mathrm{sec}] .
\end{gathered}
$$

The structure of a heating system model, corresponding to equation system (1), is shown at Fig. 1. 


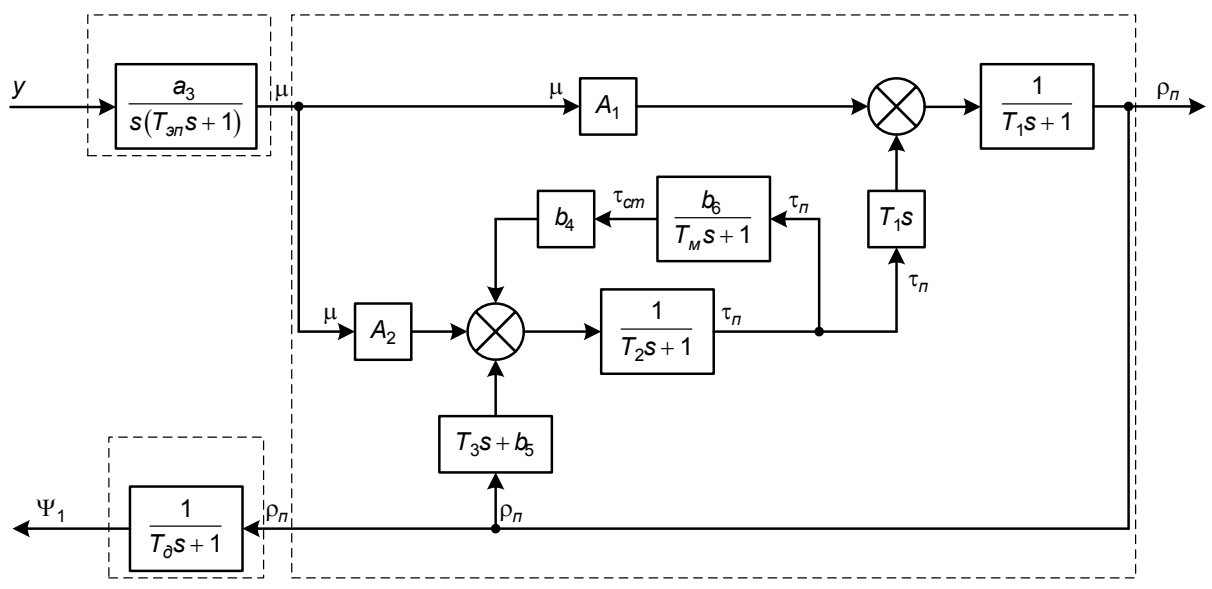

Fig. 1. Block diagram of the control object

Further we will consider the mathematical model in the form of transfer functions:

$$
\begin{gathered}
W_{\rho_{n} y}(s)=\frac{\rho_{n}(s)}{y(s)}=\frac{b_{2}^{o} \cdot s^{2}+b_{1}^{o} \cdot s+b_{0}^{o}}{a_{3}^{o} \cdot s^{3}+a_{2}^{o} \cdot s^{2}+a_{1}^{o} \cdot s+a_{0}^{o}} \cdot \frac{a_{3}}{s\left(T_{\ni n} s+1\right)}, \\
W_{\Psi_{1} y}(s)=\frac{\Psi_{1}(s)}{y(s)}=\frac{1}{T_{\partial} s+1} \cdot \frac{b_{2}^{o} \cdot s^{2}+b_{1}^{o} \cdot s+b_{0}^{o}}{a_{3}^{o} \cdot s^{3}+a_{2}^{o} \cdot s^{2}+a_{1}^{o} \cdot s+a_{0}^{o}} \cdot \frac{a_{3}}{s\left(T_{\ni n} s+1\right)},
\end{gathered}
$$

where its coefficients are determined by following:

$$
\begin{gathered}
b_{0}^{o}=A_{1} \cdot\left(1-b_{4} \cdot b_{6}\right) ; \\
b_{1}^{o}=A_{2} \cdot T_{1}+A_{1} \cdot\left(T_{2}+T_{\mathcal{M}}\right) ; \\
b_{2}^{o}=T_{M} \cdot\left(A_{1} \cdot T_{2}+A_{2} \cdot T_{1}\right) ; \\
a_{0}^{o}=1-b_{4} \cdot b_{6} ; \\
a_{1}^{o}=\left(1-b_{4} \cdot b_{6}-b_{5}\right) \cdot T_{1}+T_{2}+T_{\mathcal{M}} ; \\
a_{2}^{o}=\left(\left(1-b_{5}\right) \cdot T_{M}+T_{2}-T_{3}\right) \cdot T_{1}+T_{2} \cdot T_{\mathcal{M}} ; \\
a_{3}^{o}=T_{1} \cdot T_{\mathcal{M}} \cdot\left(T_{2}-T_{3}\right) .
\end{gathered}
$$

It is required to implement a discrete control law that provides the synthesized closedloop control system with dynamic indicators corresponding to an aperiodic transient process with a control time of no more than 35 seconds (stated from equipment characteristics and efficiency condition, [1]).

\section{Control synthesis}

The peculiarity of the control object is that the heating process of the live-steam-pipeline is performed in four stages, at each of which the numerical values of the parameters (2) are changing. That can complicate the selection process for the governor, which capable provide required system performance in all operating modes and stages of steam pipeline heating. 
There are a lot of works [3-6] where an approach is proposed that allows us to build a closed-loop control system with the properties of a given reference model. Such control algorithms also have robust properties [7]. The main idea of this approach [4, 7] is in control making based on the functional minimization condition, that is used here as the minimum energy speed up the process which is defined by the expression:

$$
J(y)=\frac{1}{2}\left[\ddot{\rho}_{\ni}(t)-\ddot{\rho}(t, y)\right]^{2},
$$

here $\rho(t, y)$ - output signal of the closed system we build; $\ddot{\rho}_{\ni}(t)$ - output signal of the reference model (etalon system), which parameters are the best fit for the required performance.

To find the control signal $y(t)$ a gradient method is used:

$$
\frac{d}{d t} y(t)=\lambda \frac{d}{d y} J(y), \lambda=\text { const } .
$$

Taking a second-order model as a reference (etalon), after the corresponding calculations that can be found in [5], the desired control $y(t)$ will be defined by the expression

$$
y(t)=K\left\{\alpha_{0} \cdot \int_{0}^{t}\left[\rho_{n}^{*}(t)-\rho_{n}(t)\right] d t-\alpha_{1} \cdot \rho_{n}(t)-\dot{\rho}_{n}(t)\right\} .
$$

Here $\rho_{n}^{*}(t)$ - changing steam pressure in pipeline we required.

As a result, the block diagram of a closed-loop control system will be as shown at Fig. 2.

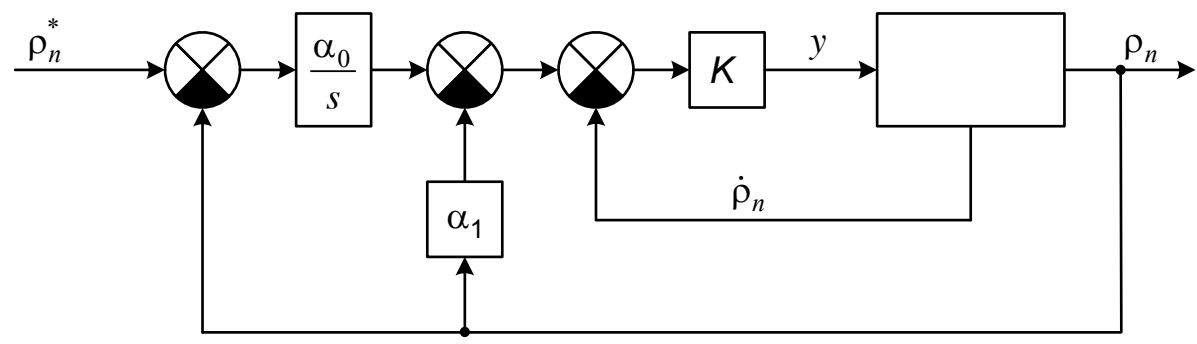

Fig. 2. Block diagram of a closed-loop control system

So, to perform a control we need an information about $\rho_{n}(t)$ и $\dot{\rho}_{n}(t)$. Farther if we decided to apply control algorithm of a discreet type, so the derivative of the output signal will be found from the known output. Therefore, the block diagram of a closed-loop control system of heating of main live-steam-pipeline can be represented as shown at Fig. 3. 


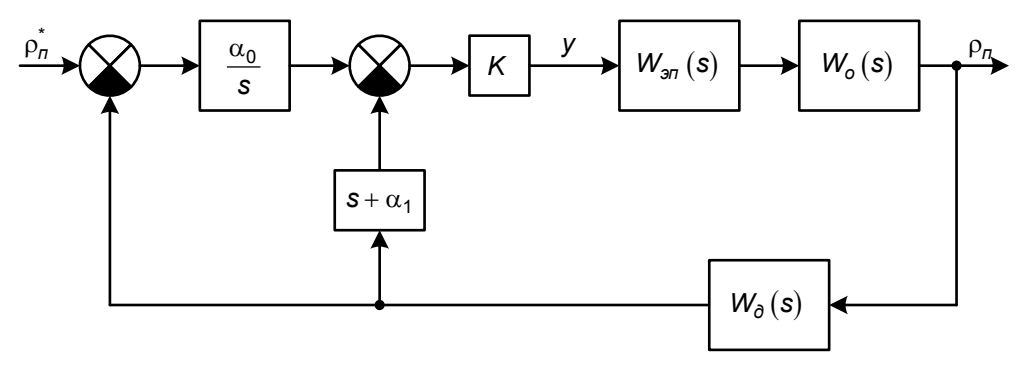

Fig. 3. Block diagram of a closed-loop control system

It only remains to determine the parameters $\alpha_{0}$ and $\alpha_{1}$ of the reference model, as well as the numerical value of the gain $K$ in the direct loop.

\section{Reference model (etalon)}

As we need to provide an aperiodic transition process of an output along with not more than second-order of a reference model, so we consider as a reference model the second-order unit with a transfer function:

$$
W_{\ni}(s)=\frac{1}{(T \cdot s+1)^{2}},
$$

the transition process of which will be close to this:

$$
h(t)=1-\left(1+\frac{1}{T} \cdot t\right) \cdot e^{-\frac{1}{T} \cdot t} .
$$

Knowing the control time $t_{\mathrm{y}}$, it is easy to find the value of the time constant $T$.

Since the transfer function should be set as [3]:

$$
W(s)=\frac{\alpha_{0}}{s^{2}+\alpha_{1} \cdot s+\alpha_{0}},
$$

then by equating the corresponding coefficients of the transfer functions (8) and (6), we can find the numerical values of the parameters $\alpha_{0}$ и $\alpha_{1}$.

\section{Results}

Now let's explore the effect of the gain value $K$ in a direct circuit on the dynamics of the closed-loop control system we got. We have the following system of equations after the Laplace transform:

$$
\left\{\begin{array}{l}
y(s)=K \cdot\left(\left(\rho_{n}^{*}(s)-W_{\partial}(s) \cdot \rho_{n}(s)\right) \cdot \frac{\alpha_{0}}{s}-\left(s+\alpha_{1}\right) \cdot W_{\partial}(s) \cdot \rho_{n}(s)\right) \\
\rho_{n}(s)=W_{o}(s) \cdot W_{\ni n}(s) \cdot y(s) .
\end{array}\right.
$$

From the last system we get: 


$$
\begin{gathered}
W_{\rho}(s)=\frac{\alpha_{0} K W_{o}(s) W_{\text {э }}(s)}{s+K\left(s^{2}+\alpha_{1} s+\alpha_{0}\right) W_{\partial}(s) W_{o}(s) W_{\text {эn }}(s)} \\
W_{y}(s)=\frac{\alpha_{0} K}{s+K\left(s^{2}+\alpha_{1} s+\alpha_{0}\right) W_{\partial}(s) W_{o}(s) W_{\text {эn }}(s)}
\end{gathered}
$$

If we represent the transfer functions of the elements as a rational fractions

$$
W_{\ni n}(s)=\frac{B_{\ni n}(s)}{A_{\ni n}(s)} ; W_{\partial}(s)=\frac{B_{\partial}(s)}{A_{\partial}(s)} ; W_{o}(s)=\frac{B_{o}(s)}{A_{o}(s)},
$$

here $W_{\text {эn }}(s), W_{\partial}(s)$ и $W_{o}(s)$ - transfer functions of the electric drive, of the sensor and of the steam pipeline. The expressions (10) could be rewritten as:

$$
\begin{gathered}
W_{\rho}(s)=\frac{\alpha_{0} K A_{\partial}(s) B_{o}(s) B_{э n}(s)}{s A_{\partial}(s) A_{o}(s) A_{э n}(s)+K\left(s^{2}+\alpha_{1} s+\alpha_{0}\right) B_{\partial}(s) B_{o}(s) B_{э n}(s)} ; \\
W_{y}(s)=\frac{\alpha_{0} K A_{\partial}(s) A_{o}(s) A_{э n}(s)}{s A_{\partial}(s) A_{o}(s) A_{э n}(s)+K\left(s^{2}+\alpha_{1} s+\alpha_{0}\right) B_{\partial}(s) B_{o}(s) B_{э n}(s)} .
\end{gathered}
$$

Obviously, if $K \rightarrow \infty$ the transfer function above is transformed in

$$
W_{\rho}(s)=\frac{\alpha_{0} A_{\partial}(s)}{\left[s^{2}+\alpha_{1} s+\alpha_{0}\right] B_{\partial}(s)} .
$$

However, in this case the control transfer function $W_{y}(s)$ will be

$$
W_{y}(s)=\frac{\alpha_{0} A_{\partial}(s) A_{o}(s) A_{\ni n}(s)}{\left(s^{2}+\alpha_{1} s+\alpha_{0}\right) B_{\partial}(s) B_{o}(s) B_{\ni n}(s)},
$$

where the numerator order is more than denominator one, i.e. control algorithm becomes fully determined by derivatives, that can often lead to unstable closed-loop control system [5]. Thus an acceptable values of the $K$ - coefficient it is possible to find from a stableclosed-loop-system condition. Such closed-loop-system simulation results with variable $K$ coefficient are shown at Figs. 4 and 5. 

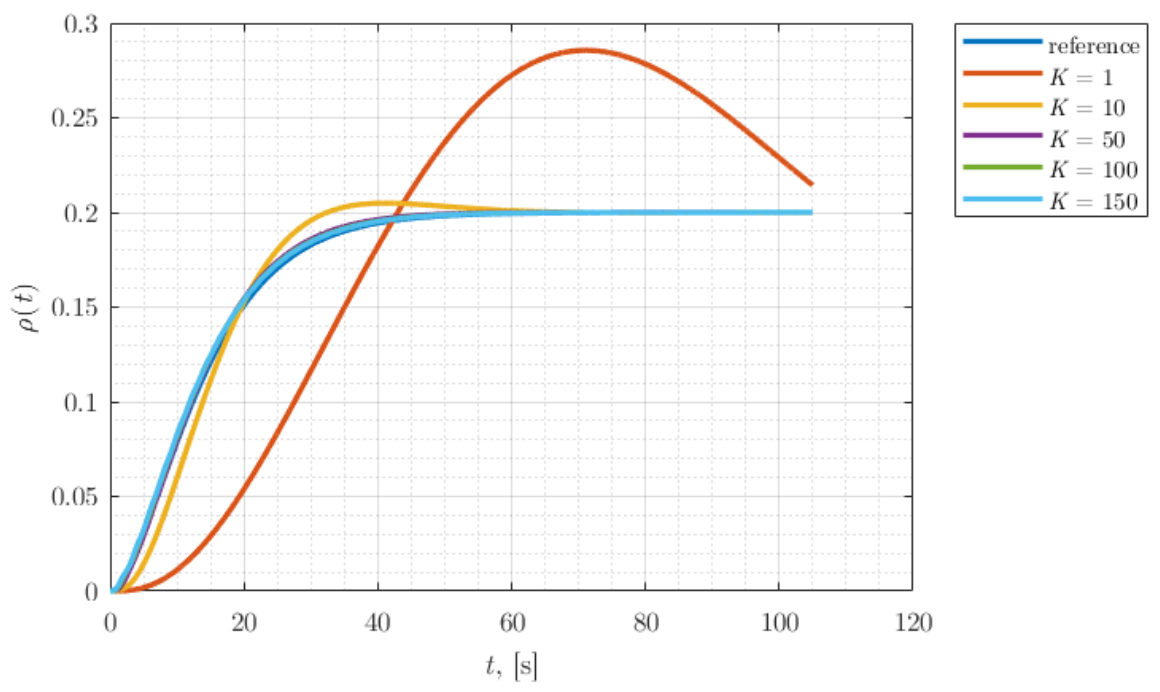

Fig. 4. An output signal in relation to $K$ - coefficient

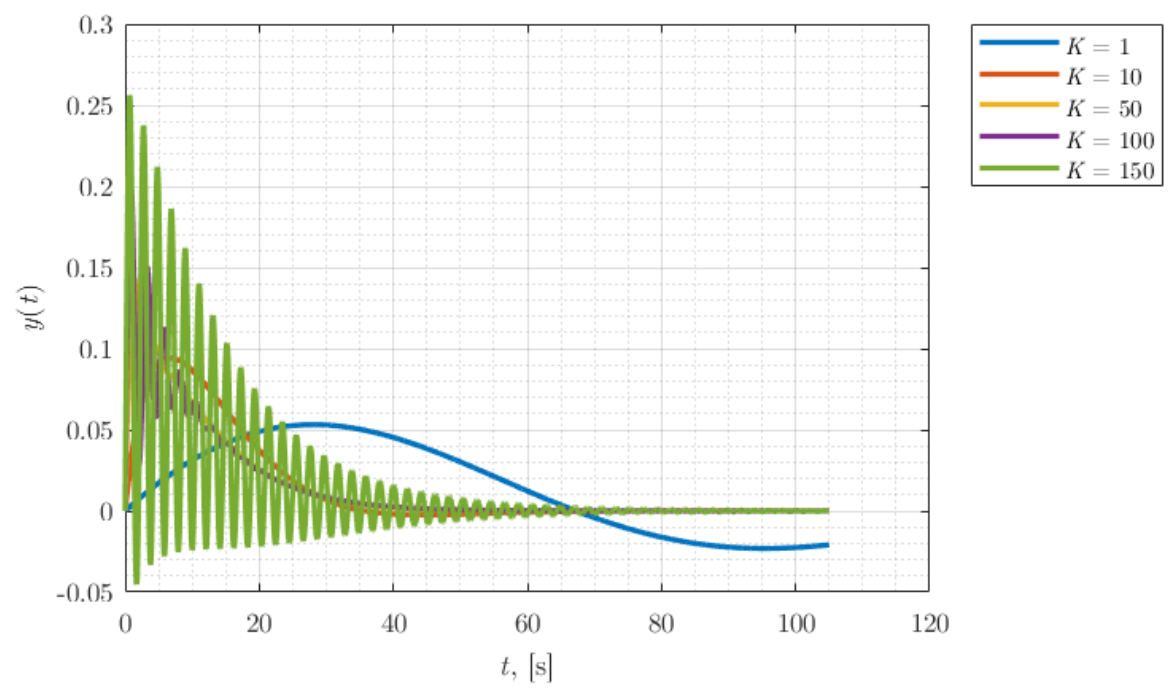

Fig. 5. Control signal in relation to $K$ - coefficient

The following conclusion can be drawn from the analysis of the influence of the $K$ coefficient value on the dynamics of the system: as the value $K$ increases, the error between the actual output signal and the specified reference signal decreases, but at the same time, the oscillation of the control signal in the transient mode increases.

Therefore, it is necessary to find a compromise between the requirement of the best match of the output signal with the specified reference signal and reducing control signal fluctuations in the transient mode.

The following criterion for this can be considered: 


$$
J(K)=\left[\int_{0}^{T}\left\{k_{\rho} \cdot\left(\rho_{\ni}(t)-\rho(t, K)\right)^{2}+k_{y} \cdot y^{2}(t, K)\right\} d t\right]^{\frac{1}{2}},
$$

here $k_{\rho}$ и $k_{y}$-weight factors, with the upper limit of the integral $T=3 t_{y}$.

The dependence graph of this criterion from $K$ with $k_{\rho}=k_{y}=1$ is shown below (fig. $6)$.

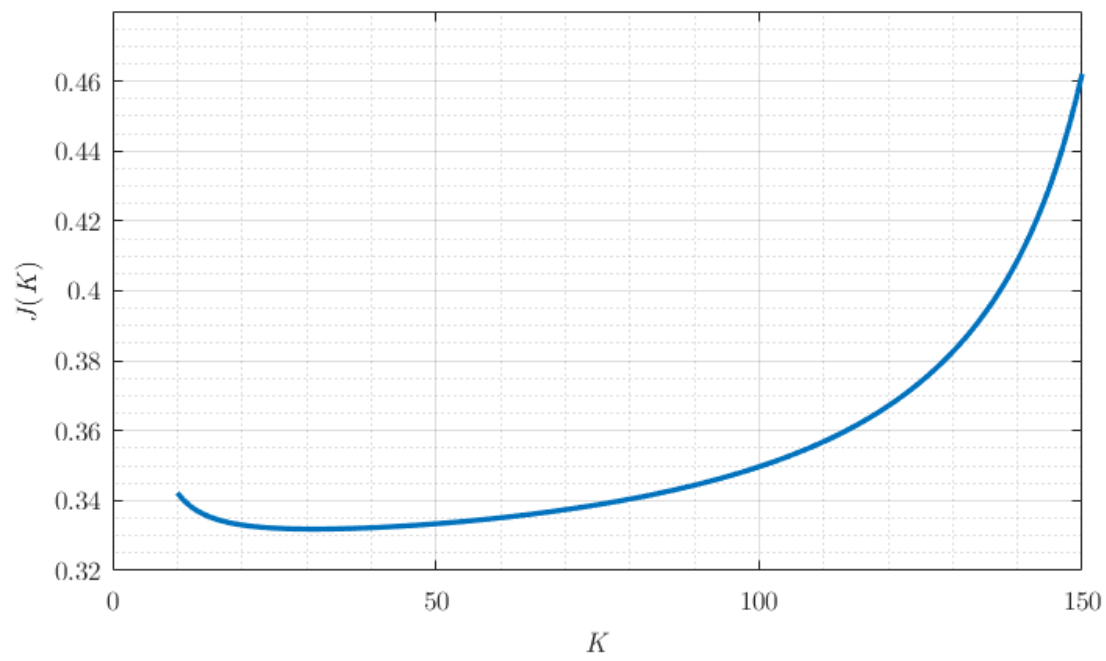

Fig. 6. The dependence graph of criterion $J$ (11) from $K$ - coefficient

The minimum of this criterion $J$ is achieved when the $K$ value is near to 31 , that is the $K_{\text {optimal }}$. For this $K$ - coefficient we obtain following graphs for the output and control signals, below (Fig. 7,8)

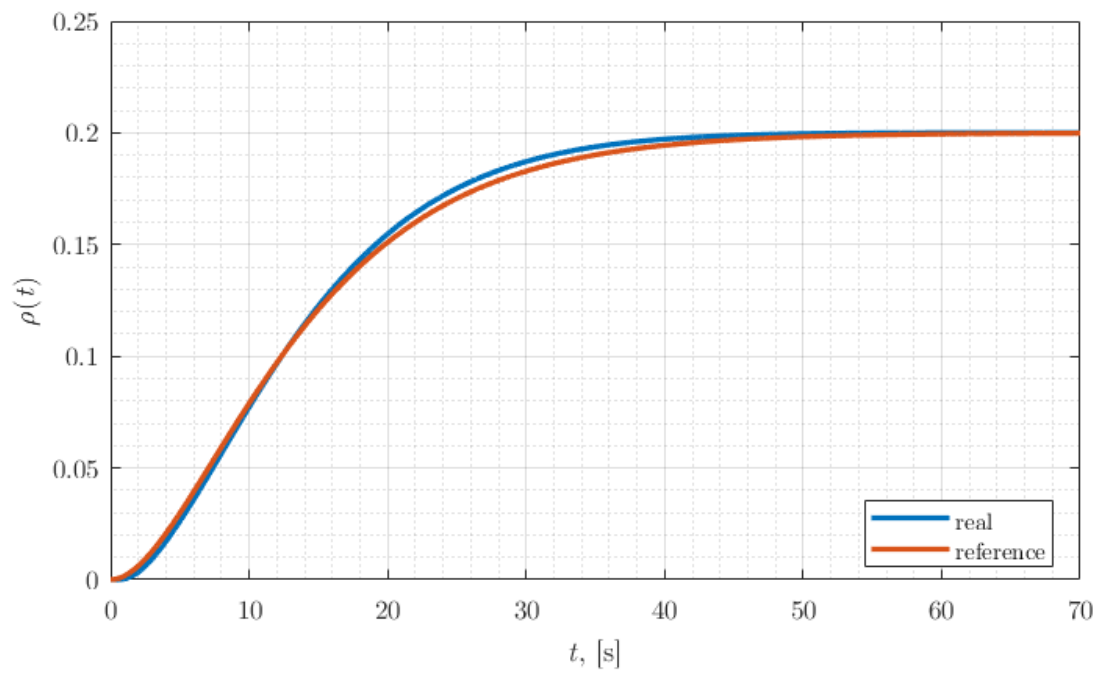

Fig. 7. Graph of the output signal at $K$ - optimal value 


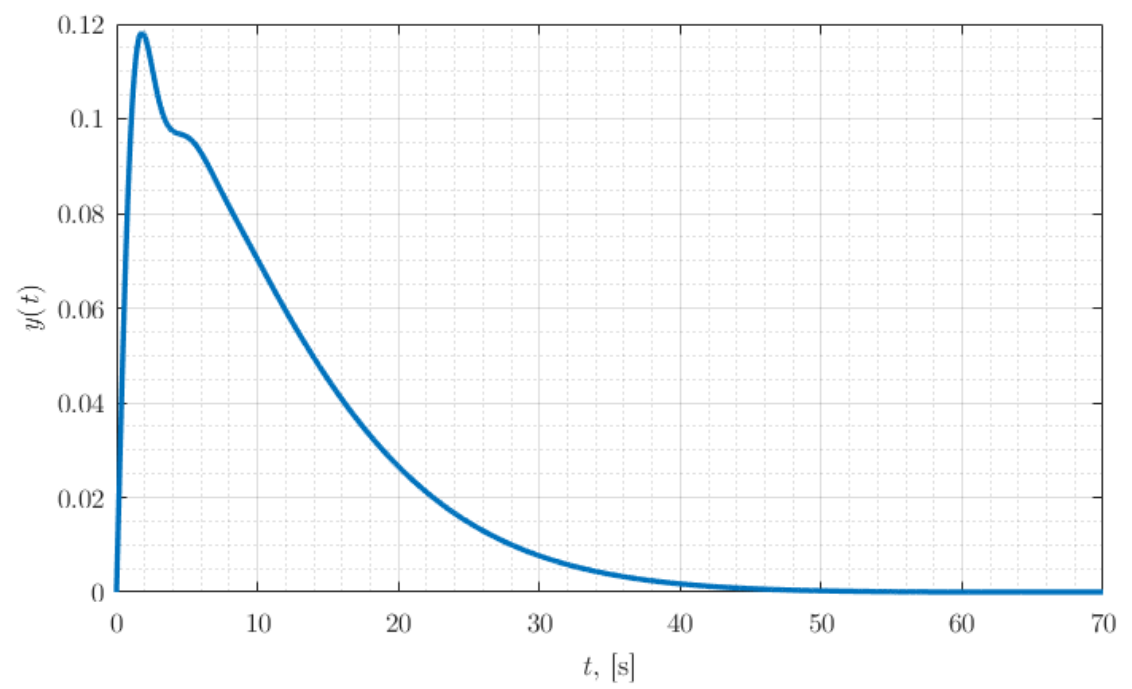

Fig. 8. Graph of the control signal at $K$ - optimal value

Heating process of the live-steam-pipeline is carried out in several stages, each of which has different numerical values of the control object parameters. Therefore the dynamic investigations of the control system built were carried at various operate modes; the results are shown below (figs. 9-10).

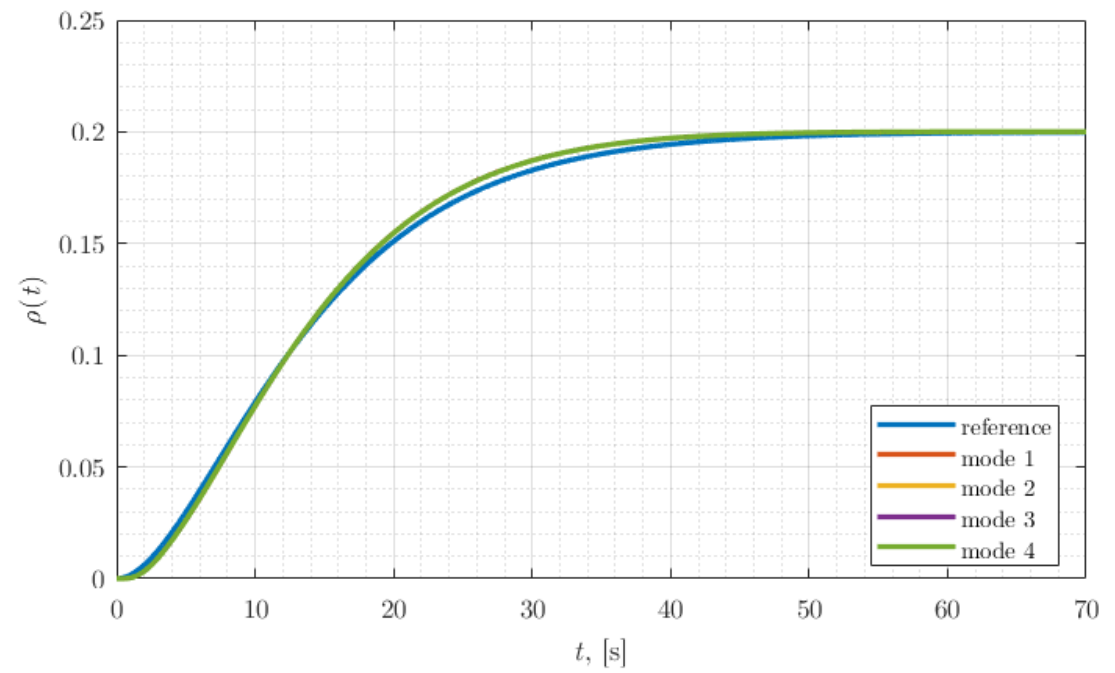

Fig. 9. Output signal graphs for the various operate modes 


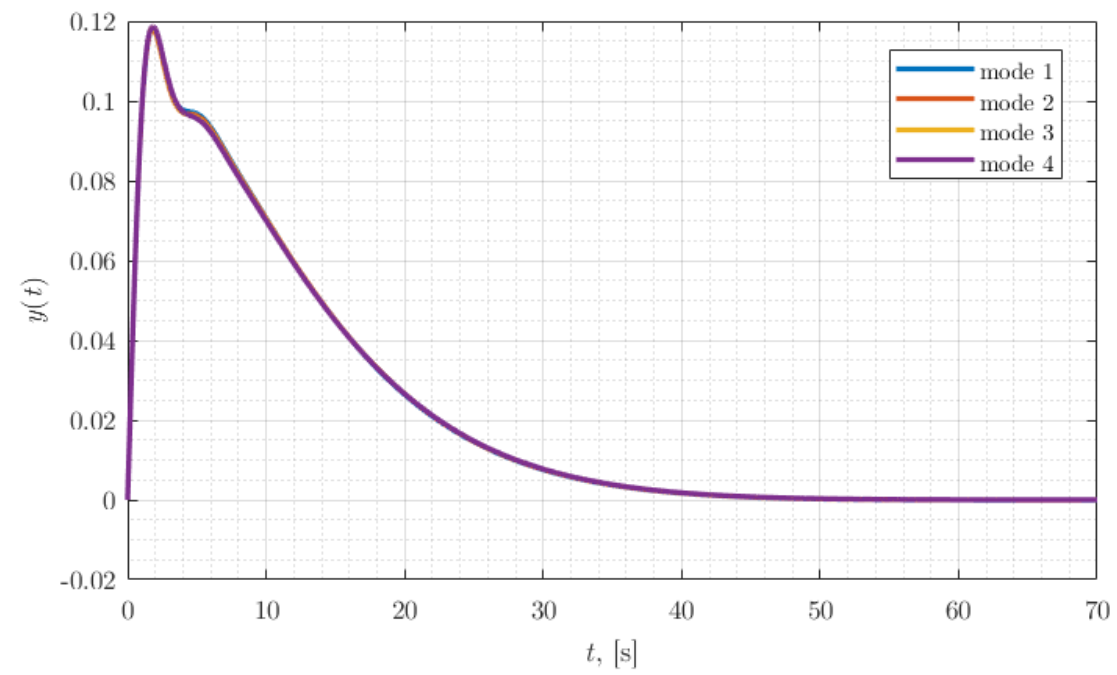

Fig. 10. Control signal graphs for the various operate modes

Thus, the constructed control algorithm allows you to provide the required dynamic performance of a closed control system in various operating modes.

Since a continuous control formation algorithm is already known (see Fig. 3), it is possible to obtain a discrete control algorithm based on it, using, for example, inverse difference method [6] or Tusten's substitution [8]. Below at fig. $11(a, b)$ the results of modeling the closed-loop discrete-continuous control system for heating a live-steam-pipeline are presented.

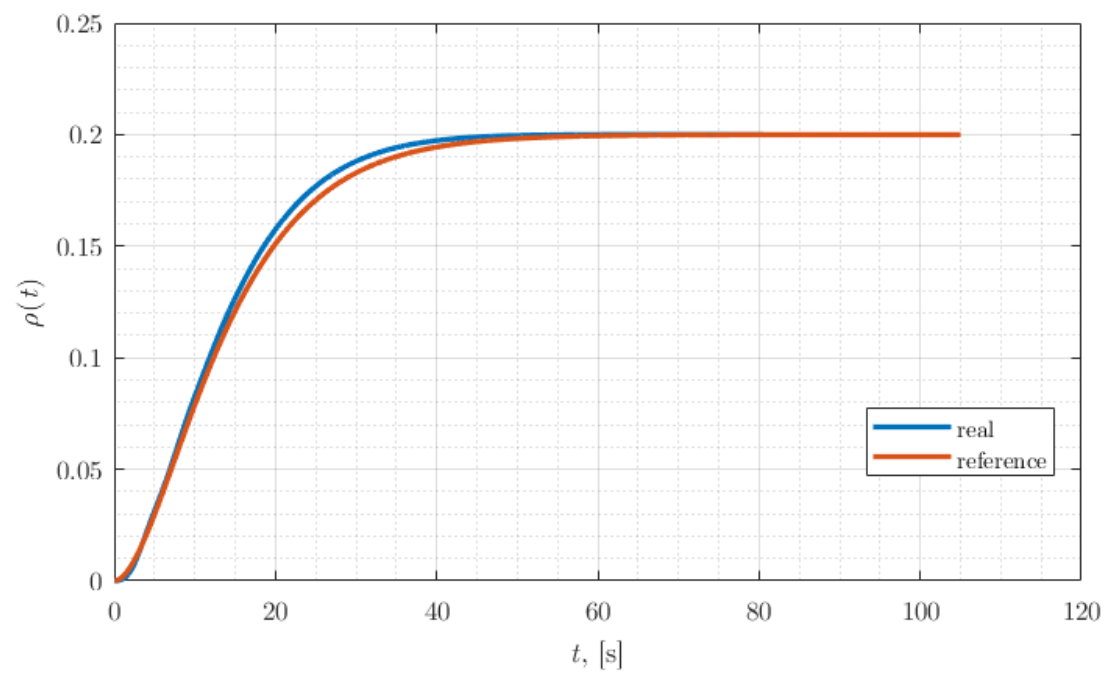

a) output signal 


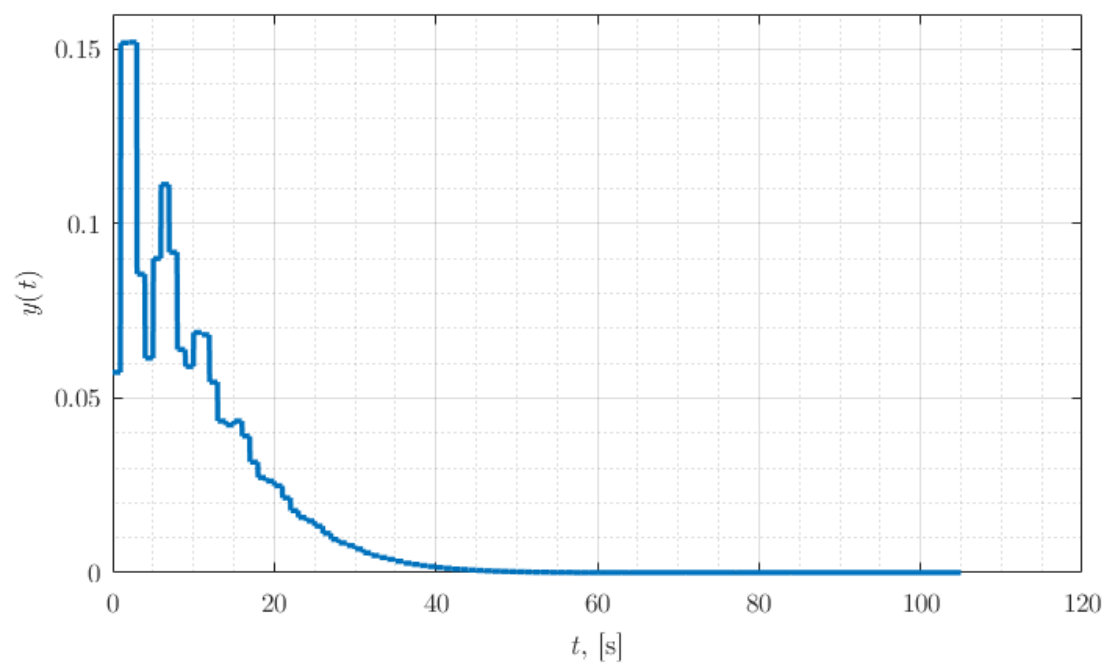

b) control signal

Fig. 11. Output and control signal graphs for the closed-loop discrete-continuous control system build

Thus, the approach to constructing the control algorithm described in $[4,9,10]$ and above allowed us to synthesize a discrete control formation algorithm that provides the required quality performance for a closed-loop discrete-continuous control system in all operating modes.

\section{References}

1. V.I. Kiryuhin, N.M. Taranenko, E.P. Ogurtzova, Low-power steam turbines of KTW, (Moscow: Energoatomizdat, 1987), p. 216.

2. D.N. Popov, Dynamics and regulation of hydraulic and pneumatic systems (Moscow: Mechanical Engineering, 1976), p. 424.

3. P.D. Krutko, Inverse problems of dynamics in the theory of automatic control (Moscow: Mechanical Engineering, 2004), p. 576.

4. P.D. Krutko, Inverse problems of the dynamics of controlled systems: linear models (Moscow: Science. The main edition of the physic-mathematical literature, 1987), p. 304.

5. P.D. Krutko, Inverse problems of the dynamics of controlled systems: nonlinear models (Moscow: Science. Ch. ed. physic-mat. lit., 1988), p. 328.

6. G. Calafiore, F. Dabbene, Probabilistic and Randomized Methods for Design under Uncertainty (London: Springer-Verlag, 2006), p. 220.

7. Matthew C. Turner, Declan G. Bates (Eds.), Mathematical Methods for Robust and Nonlinear Control (Springer, Verlag, 2007).

8. Ben-Israel, T.N.E. Greville, Generalized Inverse: Theory and Applications (NewYork: Springer Verlag, 2003), p. 400.

9. P. Khargonekar, A. Tikku, Randomized algorithms for robust control analysis and synthesis have polynomial complexity, In Proceedings IEEE Conference on Decision and Control, pp. 3470-3475 (1996).

10. P. Gahinet, P. Apkarian, A Linear Matrix Inequality Approach to Control, Int. J. Robust and Nonlinear Contr., pp. 421-448 (1994). 